\title{
Near-Death Experiences and the Theory of the Extraneuronal Hyperspace
}

\author{
Linz Audain, J.D., Ph.D., M.D. \\ George Washington University \\ The Mandate Corporation, Washington, $D C$
}

ABSTRACT: It is possible and desirable to supplement the traditional neurological and metaphysical explanatory models of the near-death experience (NDE) with yet a third type of explanatory model that links the neurological and the metaphysical. I set forth the rudiments of this model, the Theory of the Extraneuronal Hyperspace, with six propositions. I then use this theory to explain three of the pressing issues within NDE scholarship: the veridicality, precognition and "fear-death experience" phenomena.

Many scholars who write about near-death experiences (NDEs) are of the opinion that explanatory models of the NDE can be classified into one of two types (Blackmore, 1993; Moody, 1975). One type of explanatory model is the metaphysical or supernatural one. In that model, the events that occur within the NDE, such as the presence of a tunnel, are real events that occur beyond the confines of time and space. In a second type of explanatory model, the traditional model, the events that occur within the NDE are not at all real. Those events are merely the product of neurobiochemical activity that can be explained within the confines of current neurological and psychological theory, for example, as hallucination.

In this article, I supplement this dichotomous view of explanatory models of the NDE by proposing yet a third type of explanatory model: the Theory of the Extraneuronal Hyperspace. This theory represents a

Linz Audain, J.D., Ph.D., M.D., is a Resident in Internal Medicine at George Washington University, and Chief Executive Officer of The Mandate Corporation. The author is grateful for comments he has received from Dr. Adnan Eldadah. Requests for reprints should be addressed to Dr. Linz Audain, The Mandate Corporation, 4410 Massachusetts Avenue, Box 252, Washington D.C. 20016. 
combination of the two types of explanatory models in that it relies on current neurobiological concepts yet attempts to link them to the possibility of the existence of a hyperspace. The reader should be forewarned that the Theory of the Extraneuronal Hyperspace that I offer below is not at all exhaustive. Rather, it is offered merely as the beginning of what I hope will be a long debate within the scientific community as to the nature of the interaction that exists between the neurological and the metaphysical.

Accordingly, I have organized this article as follows. First I will discuss the definition and explanatory models of the NDE. Next, I will discuss three phenomena that need to be explained by any explanatory model of the NDE: the veridicality, precognition, and "fear-death experience" phenomena. Finally, I will offer and apply my Theory of the Extraneuronal Hyperspace.

\section{Definition and Explanatory Models of the NDE}

\section{Definition of the NDE}

Of the many current definitions of the NDE, there are three that are of particular relevance to this article. A first definition is Raymond Moody's (1975) characterization of the 15 elements of the typical NDE. That definition is relevant to the extent that the issue under discussion in this article is whether or not these 15 elements described by Moody, such as a ringing noise and dark tunnel, are real events or merely the products of the imagination.

A second relevant definition is Kenneth Ring's (1980) five-stage model of the NDE. The issue once again is whether some of the stages described by Ring, such as the out-of-body stage, are real or imagined events. Finally, Bruce Greyson's (1985) typology of NDEs is relevant. Greyson proposed that there are three possible types of NDE: those dominated by transcendental features, affective features, or cognitive features. Most relevant to this article is the question of the extent to which the transcendental features, such as seeing mystical beings, and cognitive features, such as a life review, are real experiences.

\section{Explanatory Models of the NDE}

Traditional models. Traditional models of the NDE abound. A first and popular hypothesis is the dying brain hypothesis (Blackmore, 1993), or, when the cause of death is hypoxia, the hypoxic brain hypothesis 
(Moody, 1975). Simply put, both hypotheses maintain that NDEs are not real experiences, but are merely the natural result of a dying brain. A second hypothesis, the pharmacological hypothesis (Moody, 1975), maintains that the NDE is the result of drugs that were administered or taken by the individual. A third hypothesis, the hallucination hypothesis (Moody, 1975), points to the similarities between the NDE and certain kinds of hallucinations, such as autoscopic hallucinations, in which individuals are able to see a visual image of themselves from the neck up.

Several authors have recently argued that the NDE represents memories that are activated by the stress of being near death. Melvin Morse, David Venecia, and Jerrold Milstein (Morse, Venecia, and Milstein, 1989) have argued that these memories are genetically coded. JeanPierre Jourdan (1994) has argued that the stress of being near death results in the blockage of those receptors responsible for the formation of new memories. Consequently, internal memories are awakened, resulting in the experience that is the NDE. Finally, Juan Gómez-Jeria and Juan Saavedra-Aguilar (1994) have argued that prior memories combine with the information contained in near-death stimuli to result in a credible "story" that is told by the subject as an NDE.

Moody (1975) discussed and, in some cases, refuted a number of the preceding hypotheses and explanatory models. Nevertheless, I believe that research on traditional explanatory models of the NDE is valuable research that should continue. Having said that, it seems to me clear that the real challenge faced by any explanatory model of the NDE is to address the three anomalous phenomena that I will discuss below.

Metaphysical models. Two decades before Moody named the NDE, Carl Gustav Jung (1955) confronted with such an experience in one of his patients, speculated on the possibility that thoughts and perceptions might have a separate metaphysical existence. Indeed, since the time of Jung's writing, there have been several scholars who have speculated that the out-of-body experience occurs outside the confines of our threedimensional existence (Greene, 1983b); and some scholars have argued that the NDE occurs outside of the confines of our three-dimensional existence (Ring, 1980, 1982; Sabom, 1982). Ring (1980), in particular, has argued that at death, consciousness occupies a fourth dimension. Kenneth Arnette $(1992,1995)$ has argued that the tunnel in NDEs may very well be a cosmological wormhole, through which experiencers travel to another universe in which three-dimensional conceptions of time and space are no longer valid. Deno Kazanis (1995) has argued 
that the mysticism of the NDE is simply a reflection of cosmological dark matter.

Perhaps the author who has been most explicit in modeling a hyperspatial approach to NDEs is Gordon Greene. In a series of articles, Greene (1980, 1981, 1983a, 1983b, 1999; Greene and Krippner, 1990) has set forth a topological model of the relationship that exists between the third dimension and higher dimensions. He has used this model to comment on the nature of the life review within the NDE (Greene, 1981 ), the experience within the NDE of simultaneously possessing two bodies (Greene, 1983a), and the separation of consciousness from the physical body in NDEs (Greene, 1999).

In his hyperspatial analysis of the life review, Greene wrote that the motivation for his analysis was that "any purely neurological theory cannot account for the paranormal effects surrounding some NDE life reviews" $(1981$, p. 120$)$. He argued further that the present challenge for near-death research is to understand "the laws of topological transformation between space and hyperspace" (p. 121). Greene then went on to suggest that when a three-dimensional being achieves sufficient "depth" in hyperspace, and time achieves a dimension of space, then both past and future may be seen. This phenomenon explains both the life review and the phenomenon of precognition, in which a near-death experiencer returns with predictions about the future. Presciently and insightfully, Greene ended that article by speculating that the neurological and hyperspatial explanations of the NDE were not necessarily mutually exclusive. He wrote: "It may be that [the two explanations] account for the vision on different levels" (p. 129). I shall have more to say below about Greene's speculation about a possible relationship between the neurological and hyperspatial explanations of the NDE; but first I will consider the nature of the veridicality, precognition, and "fear-death experience" phenomena, three anomalies that pose a remarkable challenge to traditional explanatory models of the NDE.

\section{The Veridicality, Precognition, and Fear-Death Experience Phenomena}

\section{The Veridicality Phenomenon}

The veridicality phenomenon arises when near-death experiencers return from their NDEs with veridical or truthful accounts of things, processes, or people that they observed during the time that they were presumably dead, near death, or, at a minimum, unconscious. For 
example, Ring (1980) gave an account of a woman with lifelong blindness who discussed the resuscitation equipment and procedures that she observed during her NDE. Blind from birth, she could not possibly have had personal knowledge of these things. Indeed, accurate observations by the blind constitute some of the most objective evidence for the existence of the veridicality phenomenon, in that it is difficult, if not impossible, to argue that the blind individual is fabricating or prevaricating about things previously seen. Ring has continued his work in identifying these kinds of cases (Ring and Cooper, 1997; Ring and Lawrence, 1993). Cases of veridical observations have also been discussed by others (Moody and Perry, 1988).

The veridicality phenomenon presents the traditional explanatory model of the NDE with a significant problem indeed. Consider, for example, the proposition that the NDE represents the activation of previously genetically encoded memories (Morse, Venecia, and Milstein, 1989). The problem that such a theory confronts is that genes were encoded at least one generation before the resuscitation technology was developed. It becomes difficult therefore to explain how NDErs can observe resuscitation technology and procedures that had not even been discovered at the time that their genes were encoded. Other traditional explanatory models that take the NDE-as-memory approach (GómezJeria and Saavedra-Aguilar, 1994; Jourdan, 1994) confront a similar temporal problem of how the memory of the event could possibly precede the actual occurrence of the event.

\section{The Precognition Phenomenon}

The precognition phenomenon arises when some NDErs are able to make predictions about the future after their experience. A dramatic example of this phenomenon was seen in the case of Dannion Brinkley, one of Moody's NDE subjects. Brinkley returned from his NDE with predictions about the Persian Gulf War and the fall of the Soviet Union years before those events actually came to pass (Brinkley and Perry, 1994). Others (Lundahl, 1993; Ring, 1982) have discussed the precognition phenomenon in greater detail.

Traditional explanatory models of the NDE confront a different sort of temporal problem in their attempts to explain the precognition phenomenon. In the case of the veridicality phenomenon, the issue concerns how someone can have a memory of an event that is happening in the present. In the case of the precognition phenomenon, there is no possible memory or other traditional frame of reference; the individual is 
actively making a prediction about an event that has not even occurred. Even within the traditional model, it sounds remarkably unpersuasive to argue that the individual's active prediction is based on the memory of an event that has not yet occurred.

\section{The Fear-Death Experience Phenomenon}

Finally, the "fear-death experience" phenomenon is based on a study by Ian Stevenson, Emily Cook, and Nicholas McClean-Rice (1989-1990), in which they reported that 83 percent of hospital patients with NDEs subjectively believed that they were near death. However, only 45 percent of those hospital patients were actually objectively near death, while 55 percent were not. Stevenson, Cook, and McClean-Rice referred to the latter group of patients as individuals with "fear-death experiences." Others (Floyd, 1996; Gabbard and Twemlow, 1991) have studied, corroborated, and expanded upon this phenomenon originally identified by Stevenson, Cook, and McClean-Rice.

In contrast to their explanation of the veridicality and precognition phenomena, traditional explanatory models of the NDE are on surer footing in explaining the fear-death experience. Specifically, the emphasis of traditional models on the stress of being near death (Morse, Venecia, and Milstein, 1989) is consistent with the fear-death experience's emphasis on the fear of being near death as a critical factor. The problem that arises is that many of the aspects of the conventional NDE, such as precognition (Floyd, 1996), are also present in fear-death experiences. Accordingly, the difficulties that are encountered with traditional explanations of those aspects of the NDE are also present with traditional explanations of the fear-death experience.

In view of these three anomalous phenomena and the efforts of the traditional explanatory models to explain them, the question that arises is whether any insights can be gleaned from the Theory of the Extraneuronal Hyperspace, to which I now turn.

\section{NDEs and the Theory of the Extraneuronal Hyperspace}

As discussed above, there are near-death scholars who have actively considered the possibility of a hyperspatial theory of the NDE. In addition to those specific individuals, it is testimony to the openmindedness and scientific integrity of near-death researchers that many of them are calling for and engaging in discussions about the NDE that extend beyond the traditional explanatory model. 
As an affirmation of this openmindedness, reconsider Jung's suggestion that there might be in the human organism a "nervous substrate... which is absolutely different from the cerebrospinal system ... that can evidently produce thoughts and perceptions" $(1955$, p. 124). I argue that this substrate of the nervous system may very well be an extraneuronal hyperspace. Under my Theory of the Extraneuronal Hyperspace, neurotransmitters are important not only because of the effect they have in the three-dimensional realm, but because of the effect they have beyond the third dimension, in hyperspace.

\section{Six Propositions of the Theory of the Extraneuronal Hyperspace}

I propose that there are six basic propositions of the Theory of the Extraneuronal Hyperspace.

The transduction proposition. The first proposition is the transduction proposition. Specifically, neuroscientists have asked how the human brain controls thought, and this particular question has generated an impressive body of theories and empirical research. I argue that an equally plausible but unasked question is how thought controls the brain. In answering this question, I postulate that the brain is itself an organ of signal transduction. The theme of signal transduction is a powerful and recurrent one within the neurosciences. For example, the Pacinian corpuscles transduce pressure to electrical signals, while the eye transduces photons to electrical signals. It seems plausible that the signals received by the brain, a separate neural organ, are also transduced by it.

The complexity proposition. The second proposition, a complexity proposition, refers to the nature of the signals that are transduced by the brain. Although conceivably any kind of neuronal signal is a potential candidate for transduction by the brain, it seems more plausible that signals that have already been made complex within the cortex are the chief candidates for transduction by the brain. For example, neuroscientists now believe that vision involves a building up of the simple signal registered by the neurons of the retina. Although the signals move from the retina to the occipital lobe, they do not remain there, but are channeled through ganglia of increasing complexity, such as the parvocellular-blob system (Kandel, Schwartz, and Jessell, 1991).

It seems reasonable then to argue that these signals that have already been made complex are the prime candidates for becoming more complex. I shall use the term extraneuronal element or cognon to refer 
to any signal, simple or complex, that has been transduced by the brain. The extraneuronal element or cognon is the most basic unit of analysis for understanding the extraneuronal hyperspace; its closest intuitive approximation is that it is the most basic unit of a "thought." Aggregations of extraneuronal elements are, of course, possible.

The hyperspace proposition. The third proposition of this theory, the hyperspace proposition, concerns the place to which the brain transduces these extraneuronal elements or cognons and their aggregations. It seems plausible, for the following four reasons, to argue that extraneuronal elements are transduced into an energy form and a space beyond the three dimensions, that is, a hyperspace. One reason is that such a transduction from three-dimension space into the hyperspace through an Einstein-Rosen "hyperspace tunnel" is possible at the level of theoretical physics (Rucker, 1984). As noted above, some scientists have already begun to think of the NDE tunnel as a wormhole into another dimension. I suggest that comparable means of interdimensional transport exist in connection with the physical brain to allow transduction of energy or matter. A second reason to accept the hyperspace proposition is that it can explain how the massive amount of electrical information processed and received by the cortex can be stored and processed. A third reason is that a hyperspace explanation explains how those who leave their bodies in an NDE can see and hear those around them, but cannot themselves be seen or heard (Brinkley and Perry, 1994): they are, at that point, inhabitants of a dimension higher than, and subsuming, our three-dimensional world.

A fourth reason to appeal to a hyperspace explanation of consciousness is to explain the empirically observed phenomenon of time delays of consciousness. With respect to actions consciously willed by individuals, a series of experiments by different investigators have demonstrated that "conscious action takes something like a second to a second and a half before it can be effected, while ... consciousness of an external event does not seem to occur until half a second after that event has taken place" (Penrose, 1989, p. 439). The most interesting aspect of these experiments however, is that the individual does not perceive any time as having elapsed.

One possible explanation for this anomaly is that perceptions or thoughts live in hyperspace, where time is a manipulable variable. Indeed, the ratio of hyperspace time to three-dimensional time is extremely, if not infinitesimally, small, so that things that take a long time in the three-dimensional world take very little time in hyperspace. For example, the beings whom Brinkley met in hyperspace were able to 
show him his entire life in review and the future of Earth spanning more than 20 years, all in less than 30 minutes of three-dimensional Earth time (Brinkley and Perry, 1994); this point regarding the timelessness of the life review in a higher dimension was also made by Greene (1981). Accordingly, in the experiments mentioned above, volitional movement recorded by electroencephalogram (EEG) shows that three-dimensional time has elapsed, but in the consciousness, which lives in hyperspace, no time or very little time has elapsed.

The bidirectionality proposition. A fourth proposition on which I premise the Theory of the Extraneuronal Hyperspace, the bidirectionality proposition, states that there can be transduction both into and out of the extraneuronal hyperspace. Note that the hyperspace remains a part of the nervous system. It is therefore not extraneural, but merely extraneuronal, raising the possibility of not just central and peripheral nervous systems, but an extraneuronal nervous system as well.

Conceivably, there can be transduction of certain types of signals into or out of the extraneuronal hyperspace through preferred loci in the brain, for example, speech through Wernicke's area in the left hemisphere. But these are only preferred and not required loci. The Theory of the Extraneuronal Hyperspace therefore explains the empirically observed phenomenon in which humans who have had their corpus callosum surgically severed, although having no connection between the left and right hemisphere, are ultimately able to "learn to speak" with the right hemisphere (Penrose, 1989, p. 385). This is possible because it is primarily thought that preexists and controls the neurons, rather than the other way around. New hyperspace channels can therefore be forged through different loci in the brain, to make the transduction of thought out of the extraneuronal hyperspace possible.

I am willing to concede that sensory input from the outside world can alter the shape of consciousness in hyperspace. There is, after all, transduction of sensory input into the hyperspace. However, it is the nature and quality of the input that matters in that instance. Here I mean only to emphasize that, holding the nature of input constant, activity in hyperspace can affect the location through which thought is transduced out of the hyperspace into the three-dimensional brain.

The matrix proposition. A fifth proposition, the matrix proposition, concerns the manner in which the extraneuronal elements or cognons organize themselves in the extraneuronal hyperspace. I will call this organization the extraneuronal matrix, which is equivalent to "the mind." Positing the existence of an extraneuronal matrix makes possible the explanation of a number of puzzling phenomena. For example, if there 
are specialized sensory cognons within the extraneuronal matrix, then it becomes possible to explain how we can "see with our mind's eye": the explanation is that such visualization is occurring in hyperspace.

However, transducing into the three-dimensional brain what has been seen in hyperspace is a problem fraught with great physical difficulty; for example, neurons have to be resynapsed. Those rare individuals who are able to transduce out of the extraneuronal space with relative neuronal efficiency are often very productive artists. For example, my productivity as a musical composer is limited simply because the quantity and quality of music that I hear in my mind's ear far outstrips my capability to transduce it from my extraneuronal space into my three-dimensional brain; Mozart had no such problem. This explains why blind persons who have NDEs can now see: their separation from the body means that transduction out of the extraneuronal hyperspace through nonfunctioning three-dimensional neurons in the optical system is no longer necessary. As another example, anesthetized surgical patients are able to respond to the surgeon's request that they control their own blood flow simply because their minds remain functional despite the anesthetized state of their brains.

The tunnel closure proposition. A sixth proposition on which I premise the Theory of the Extraneuronal Hyperspace, the tunnel closure proposition, is that states of physical alertness correspond to the degree of closure of the Einstein-Rosen hyperspace tunnel and the amount of transduction in both directions through the hyperspace tunnel. For example, sleep and NDEs correspond to different states of limited and reversible closure of the hyperspace tunnel. Death, on the other hand, corresponds to a permanent and irreversible closure of the hyperspace tunnel, as well as an irreversible separation of the extraneuronal matrix from the physical body. At the point of death, the most recent extraneuronal matrix becomes a part of what some have called the metaconsciousness (Whitton and Fisher, 1986): evidence from regression to apparent memories of past lives suggests that the most recent extraneuronal matrix, along with prior extraneuronal matrices, may be accessible through hypnosis, although a new extraneuronal matrix is formed with each new incarnation.

\section{The Veridicality, Precognition, and Fear-Death Experience Phenomena}

Based on these six propositions of the Theory of the Extraneuronal Hyperspace, the three phenomena discussed above-veridicality, precognition, and the fear-death experience-are reduced to one question. 
That is, what is the nature of the interface between the three-dimensional body and hyperspace? The reason that the question of the interface is most significant is that the veridicality and precognition phenomena can be explained mainly by assuming the existence of an extraneuronal hyperspace. However, the mere existence of an extraneuronal hyperspace is not sufficient to explain the fear-death experience.

Specifically, precognition is possible because time is a manipulable variable in the hyperspace. Accordingly, as Greene argued (1981), when an individual reaches the appropriate level or dimension in hyperspace, he or she is able to see the past and the future, giving rise to the subjective experiences of the life review and precognition. Veridical observations are possible because the individual is actually seeing and hearing three-dimensional activity from the vantage point of hyperspace. As discussed in the matrix proposition above, the sight and sound sensory modalities remain functional, and indeed become even more functional, once they have been separated from a three-dimensional body.

With regard to the fear-death experience, I suggest that neurotransmitters play a clear role in inducing the experience. However, it is possible to integrate neurotransmitters into the Theory of the Extraneuronal Hyperspace. One possibility is that the neurotransmitters, in addition to their effect on the physical neurons of the brain, have a simultaneous extradimensional impact that cannot be detected by our present instruments. Under this explanation, the neurotransmitters make possible a temporary closure of the hyperspace tunnels, allowing a temporary separation of the extraneuronal matrix from the physical body. A second possibility is that the neurotransmitters may affect another three-dimensional object that in turn has the extradimensional effect on the hyperspace tunnels.

\section{Conclusion}

In sum, an explanation that integrates neurotransmitters into a discussion of hyperspace makes it possible to understand veridical observations, precognition, and the fear-death experience as real phenomena. It is not necessary for the theory to ignore these phenomena, or to assert that they do not really exist.

Of course, the Theory of the Extraneuronal Hyperspace, if true, has implications beyond the NDE. Consider for example, the mystery of the concussion, in which an individual who has lost consciousness after a blow to the head regains consciousness with no evidence of neuronal damage (Andreoli, Bennett, Carpenter, Plum, and Smith, 1993). One 
speculation from the Theory of the Extraneuronal Hyperspace as to why this might be so is that a three-dimensional displacement of the brain, perhaps the ventricles, may result in an extradimensional displacement, perhaps of the extraneuronal matrix, with a temporary closure of the channels of transduction and, consequently, loss of consciousness. Extradimensional realignment produces a reopening of the channels and regaining of consciousness in the three-dimensional world. Yet no damage can be observed in the three-dimensional brain because the real damage was extradimensional.

This then is work for another day. For now, I offer the Theory of the Extraneuronal Hyperspace as a beginning in many respects. But in other respects it is also a continuation, a continuation of the voices of the many of us who believe that we scientists have underestimated the role of the hyperspace in our daily lives, as well as our deaths. With this continuation then, it is my fervent hope that someday our voices will be heard not occasionally, but collectively, as a single, wonderful, and intellectually mellifluous chorus.

\section{References}

Andreoli, T. E., Bennett, J. C., Carpenter, C. C. J., Plum, F., and Smith, L. H. (Eds.). (1993). Cecil's essentials of medicine. Philadelphia, PA: Saunders.

Arnette, J. K. (1992). On the mind/body problem: The theory of essence. Journal of NearDeath Studies, 11, 5-18.

Arnette, J. K. (1995). The theory of essence. II. An electromagnetic-quantum mechanical model of interactionism. Journal of Near-Death Studies, 14, 77-99.

Blackmore, S. J. (1993). Dying to live: Near-death experiences. Buffalo, NY: Prometheus.

Brinkley, D., and Perry, P. (1994). Saved by the light: The true story of a man who died twice and the profound revelations he received. New York, NY: Villard.

Floyd, K. (1996). ECT: TNT or TLC? A near-death experience triggered by electroconvulsive therapy. Journal of Near-Death Studies, 14, 187-195.

Gabbard, G. O., and Twemlow, S. W. (1991). Do "near-death experiences" occur only near death?-Revisited. Journal of Near-Death Studies, 10, 41-47.

Gómez-Jeria, J. S., and Saavedra-Aguilar, J. C. (1994). A neurobiological model for neardeath experiences. II: The problem of recall of real events. Journal of Near-Death Studies, $13,81-89$.

Greene, F. G. (1980). A glimpse behind the life review. Theta, 8(2), 10-14.

Greene, F. G. (1981). A glimpse behind the life review. Journal of Religion and Psychical Research, 4, 113-130.

Greene, F. G. (1983a). Multiple mind/body perspectives and the out-of-body experience. Anabiosis: The Journal of Near-Death Studies, 3, 39-62.

Greene, F. G. (1983b). The out-of-body experience, extrasomatic or intrasomatic phenomenon? A non-Euclidean, higher space approach. Journal of Religion and Psychical Research, 6, 159-180.

Greene, F. G. (1999). A projective geometry for separation experiences. Journal of NearDeath Studies, 17, 151-191. 
Greene, F. G., and Krippner, S. (1990). Panoramic vision: Hallucination or bridge into the beyond? In G. Doore (Ed.), What survives? Contemporary explorations of life after death (pp. 61-75). Los Angeles, CA: Tarcher.

Greyson, B. (1985). A typology of near-death experiences. American Journal of Psychiatry, $142,967-969$.

Jourdan, J.-P. (1994). Near-death and transcendental experiences: Neurophysiological correlates of mystical traditions. Journal of Near-Death Studies, 12, 177-199.

Jung, C. G. (1955). The interpretation and nature of psyche. New York, NY: Pantheon.

Kandel, E. R., Schwartz, J. H., and Jessell, T. M. (1991). Principles of neural science. New York, NY: Elsevier.

Lundahl, C. R. (1993). Otherworld personal future revelations in near-death experiences. Journal of Near-Death Studies, 11, 171-179.

Moody, R. A. (1975). Life after life. Covington, GA: Mockingbird Books.

Moody, R. A., and Perry, P. (1988). The light beyond. New York, NY: Bantam.

Morse, M. L., Venecia, D., and Milstein, J. (1989). Near-death experiences: A neurophysiologic explanatory model. Journal of Near-Death Studies, 8, 45-53.

Penrose, R. (1989). The emperor's new mind: Concerning computers, minds, and the laws of physics. New York, NY: Oxford University Press.

Ring, K. (1980). Life at death: A scientific investigation of the near-death experience. New York, NY: Coward, McCann and Geoghegan.

Ring, K. (1982). Precognitive and prophetic visions in near-death experiences. Anabiosis: The Journal of Near-Death Studies, 2, 47-74.

Ring, K., and Cooper, S. (1997). Near-death and out-of-body experiences in the blind: A study of apparent eyeless vision. Journal of Near-Death Studies, 16, 101-147.

Ring, K., and Lawrence, M. M. (1993). Further evidence for veridical perceptions during near-death experiences. Journal of Near-Death Studies, 11, 223-229.

Rucker, R. (1984). The fourth dimension: Toward a geometry of higher reality. Boston, MA: Houghton, Mifflin.

Sabom, M. B. (1982). Recollections of death: A medical investigation. New York, NY: Harper and Row.

Stevenson, I., Cook, E. W., and McClean-Rice, N. (1989-90). Are persons reporting "neardeath experiences" really near-death? A study of the medical records. Omega 21, 45-54.

Whitton, J. L., and Fisher, J. (1986). Life between life. New York, NY: Warner Books. 